\title{
Including poroelastic effects in the linear slip theory
}

\author{
J. Germán Rubino ${ }^{1}$, Gabriel A. Castromán², Tobias M. Müller ${ }^{3}$, Leonardo B. Monachesi², \\ Fabio I. Zyserman ${ }^{2}$, and Klaus Holliger ${ }^{1}$
}

\begin{abstract}
Numerical simulations of seismic wave propagation in fractured media are often performed in the framework of the linear slip theory (LST). Therein, fractures are represented as interfaces and their mechanical properties are characterized through a compliance matrix. This theory has been extended to account for energy dissipation due to viscous friction within fluid-filled fractures by using complex-valued frequency-dependent compliances. This is, however, not fully adequate for fractured porous rocks in which wave-induced fluid flow (WIFF) between fractures and host rock constitutes a predominant seismic attenuation mechanism. In this letter, we develop an approach to incorporate WIFF effects directly into the LST for a 1D system via a complex-valued, frequency-dependent fracture compliance. The methodology is validated for a medium permeated by regularly distributed planar fractures, for which an analytical expression for the complex-valued normal compliance is determined in the framework of quasistatic poroelasticity. There is good agreement between synthetic seismograms generated using the proposed recipe and those obtained from comprehensive, but computationally demanding, poroelastic simulations.
\end{abstract}

\section{INTRODUCTION}

The seismic method is widely used for the detection and characterization of fractures, mainly because seismic waves propagating in fractured rocks show strong directional dependence and are also significantly attenuated and delayed (e.g., Peacock et al., 1994; Maultzsch et al., 2003; Clark et al., 2009). However, the explicit representation of fractures in seismic forward modeling algorithms is challenging because there is a huge length-scale contrast between typical fracture apertures and seismic wavelengths. To overcome this issue, a methodology referred to as the linear slip theory (LST) has been proposed (Schoenberg, 1980; Pyrak-Nolte et al., 1990). Therein, each fracture is replaced by an interface separating two regions of solid material representing the host rock. Although the stresses are considered to be continuous across such interfaces, the seismic effect of the fracture manifests itself as a displacement discontinuity. The displacement discontinuity vector $[\mathbf{u}]$ and the stress traction acting across the fracture $\boldsymbol{\tau} \cdot \mathbf{n}$ are linearly related through

$$
[\mathbf{u}]=\mathbf{Z} \boldsymbol{\tau} \cdot \mathbf{n},
$$

where $\boldsymbol{\tau}$ is the stress tensor, $\mathbf{n}$ is the unit normal to the fracture, and $\mathbf{Z}$ is the compliance matrix characterizing the mechanical properties of the fracture (e.g., Coates and Schoenberg, 1995). For a rotationally symmetric fracture perpendicular to the $z$-axis embedded in an elastic host rock, the compliance matrix is given by

$$
\mathbf{Z}=\left(\begin{array}{ccc}
Z_{T} & 0 & 0 \\
0 & Z_{T} & 0 \\
0 & 0 & Z_{N}
\end{array}\right)
$$

where $Z_{N}$ and $Z_{T}$ are the normal and tangential compliances of the fracture (e.g., Schoenberg, 1980). The LST allows to quantify the seismic effects of various fracture characteristics, such as the spatial distribution of fractures as well as their orientation, density, and size (e.g., Bakulin et al., 2000; Vlastos et al., 2003; Hall and Wang, 2012).

The LST can be generalized to account for seismic attenuation and dispersion through a complex-valued, frequency-dependent compliance matrix $\mathbf{Z}$ (Coates and Schoenberg, 1995). Pyrak-Nolte et al. (1990) consider discontinuities in the displacements and

\footnotetext{
Manuscript received by the Editor 26 August 2014; revised manuscript received 20 November 2014; published online 27 February 2015.

${ }^{1}$ University of Lausanne, Applied and Environmental Geophysics Group, Lausanne, Switzerland. E-mail: german.rubino@unil.ch; klaus.holliger@unil.ch.

${ }^{2}$ CONICET, Universidad Nacional de La Plata, Facultad de Ciencias Astronómicas y Geofísicas, La Plata, Argentina. E-mail: gcastroman@ fcaglp.fcaglp.unlp .edu.ar; lmonachesi@fcaglp.unlp.edu.ar; zyserman@fcaglp.unlp.edu.ar.

${ }^{3}$ Commonwealth Scientific and Industrial Research Organization, Energy Flagship, Perth, Australia. E-mail: tobias.mueller@csiro.au.

(C) 2015 Society of Exploration Geophysicists. All rights reserved.
} 
velocities across the fracture to model the viscous coupling between the two surfaces of a fluid-filled fracture. Liu et al. (2000) present an analytical expression for the complex-valued compliance of a fracture modeled as a thin continuous layer of viscous fluid or soft material. Chichinina et al. (2009) model seismic attenuation in fractured media by considering complex-valued normal and tangential compliances. In all of these studies, the imaginary part of the fracture compliance is associated with viscous friction within the fluidfilled fracture that leads to energy loss. Conversely, the host rock is approximated by an elastic solid. A more realistic scenario is to consider fractures as being embedded in a porous, permeable, and fluid-saturated host rock (e.g., Chapman, 2003). In this case, oscillatory fluid flow takes place between the fractures and the host rock in response to the applied oscillatory normal stresses and, consequently, the compliances of the fractures increase and become frequency dependent (Worthington, 2008). This oscillatory fluid flow, commonly referred to as wave-induced fluid flow (WIFF), also causes energy dissipation and, thus, seismic attenuation (e.g., Rubino et al., 2014). Moreover, Rubino et al. (2014) show that for the frequency range at which seismic attenuation due to WIFF is strong, the displacement discontinuity across fractures is complex valued and frequency dependent. These results thus indicate that this parameter may be interpreted as a proxy for attenuation and velocity dispersion in fractured porous media. At present, it is, however, not clear what the equivalent fracture compliance should be for this scenario.

In this letter, we propose a methodology to include WIFF effects in the LST for a 1D system. For simplicity, we consider a medium composed by a regular distribution of fractures and derive an analytical expression for their complex-valued, frequency-dependent normal compliances. We also develop a recipe for the generation of synthetic seismograms in fractured media based on a space-frequency domain implementation of the elastodynamic wave equation (Castromán and Zyserman, 2013), in which complex-valued frequency-dependent fracture compliances can be readily incorporated. We validate our approach by comparisons with comprehensive poroelastic simulations.

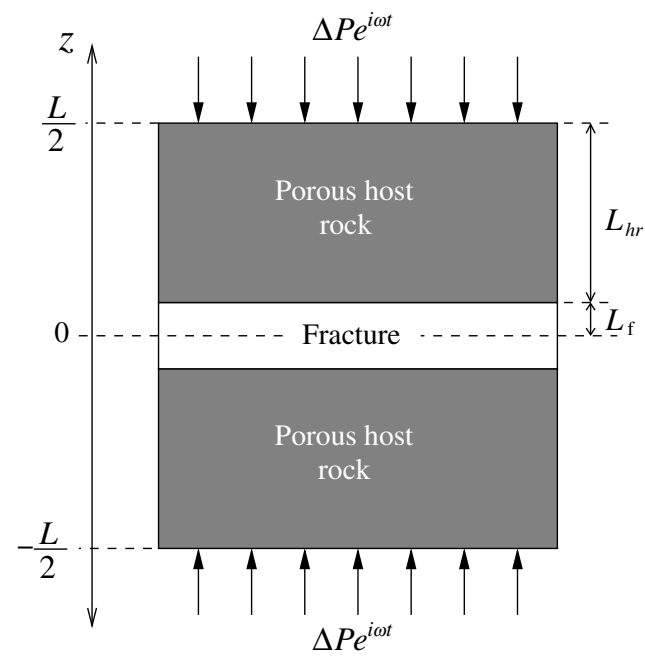

Figure 1. Schematic representation of the REV considered to study WIFF effects on fracture normal compliance.

\section{THEORY}

\section{Fractures conceptualized as thin poroelastic layers}

In analogy with the geometry analyzed by White et al. (1975), we consider a periodic 1D system of relatively thick horizontal layers of porous host rock alternating with very thin layers representing the fractures. The direction of wave propagation is along the $z$-axis and, thus, perpendicular to the fractures. The distance between consecutive fractures $L$ is assumed to be much smaller than the seismic wavelengths. Elastic scattering effects are thus negligible, whereas attenuation and velocity dispersion due to WIFF can be significant. Following Brajanovski et al. (2006), we model the seismic response of fractured rocks in the framework of Biot's (1962) theory of poroelasticity. Therein, a fracture is conceptualized as a highly compliant and highly permeable poroelastic layer. Because we are interested in computing the effects of WIFF on the displacement discontinuity produced by an applied normal stress (equation 1), we solve the $1 \mathrm{D}$ consolidation equations

$$
\begin{gathered}
\frac{\partial \tau_{z z}}{\partial z}=0 \\
i \omega \frac{\eta}{\kappa} w_{z}=-\frac{\partial p_{f}}{\partial z} .
\end{gathered}
$$

In these equations, $p_{f}$ is the fluid pressure, $\omega$ is the angular frequency, $\eta$ is the fluid shear viscosity, and $\kappa$ is the permeability, whereas $w_{z}$ denotes the relative fluid-solid displacement in the direction of the $z$-axis, and $\tau_{z z}$ is the corresponding total stress tensor component. These two equations are coupled through the stressstrain relations

$$
\begin{gathered}
\tau_{z z}=H \frac{\partial u_{z}}{\partial z}+\alpha M \frac{\partial w_{z}}{\partial z}, \\
p_{f}=-\alpha M \frac{\partial u_{z}}{\partial z}-M \frac{\partial w_{z}}{\partial z},
\end{gathered}
$$

where $u_{z}$ denotes the solid displacement in the direction of the $z$ axis. The undrained $\mathrm{P}$-wave modulus $H$, the Biot-Willis parameter $\alpha$, and the fluid storage modulus $M$ can be expressed in terms of poroelastic end-member properties (Biot, 1962).

\section{Frequency-dependent fracture compliance}

The representative elementary volume (REV) of the $1 \mathrm{D}$ periodic fracture system is shown in Figure 1. We choose the center of the REV as the origin of the $z$-axis and therefore, the positions of the upper and lower boundaries of the fracture are $z=L_{\mathrm{f}}$ and $z=-L_{\mathrm{f}}$, respectively. The thicknesses of the two embedding regions are $L_{\mathrm{hr}}$ and, thus, the total thickness of the REV is $L=2\left(L_{\mathrm{f}}+L_{\mathrm{hr}}\right)$. The $\mathrm{REV}$ is subjected to a time-harmonic compression of the form $\Delta P e^{i \omega t}$ at its top and bottom boundaries, which emulates the stress field of a monochromatic P-wave with a wavelength that is much larger than $L$. Due to the model's symmetry, fluid flow is zero at the boundaries of the REV, and no solid or fluid displacements occur at 
its center. Given the inherent 1D character of the equations, the fracture normal compliance is then given by

$$
Z_{N}(\omega)=\frac{\left[u_{z}\right]}{\tau_{z z}}=\frac{u_{z}\left(L_{\mathrm{f}}\right)-u_{z}\left(-L_{\mathrm{f}}\right)}{-\Delta P} .
$$

Solving equations 3-6 under the aforementioned boundary conditions yields

$$
Z_{N}(\omega)=Z_{N}^{\mathrm{nf}}+\frac{2 B_{\mathrm{f}}\left(B_{\mathrm{f}}-B_{\mathrm{hr}}\right)}{N_{\mathrm{f}} k_{\mathrm{f}} \operatorname{coth}\left(k_{\mathrm{f}} L_{\mathrm{f}}\right)+N_{\mathrm{hr}} k_{\mathrm{hr}} \operatorname{coth}\left(k_{\mathrm{hr}} L_{\mathrm{hr}}\right)},
$$

where $Z_{N}^{\mathrm{nf}}=2 L_{\mathrm{f}} / H_{\mathrm{f}}, N=M(1-\alpha B)$, and $k=\sqrt{i \eta \omega / N \kappa}$ is the wavenumber of Biot's slow P-wave. The parameter $B=\alpha M / H$ is the uniaxial Skempton coefficient, that is, the ratio of the fluid pressure increase to the applied stress for undrained conditions. The subscripts "f" and " $h r$ " refer to the parameters of the fracture and the host rock, respectively.

Equation 8 is the main result of this study. We observe that the fracture normal compliance consists of two components. $Z_{N}^{\text {nf }}$ is the expression used in the LST when the fracture is modeled as a thin layer of soft material and WIFF does not exist. Hence, the superscript " $n f$ ' for no-flow. The second term in equation 8 represents the WIFF component, which causes an increase of the fracture compliance as a consequence of fluid pressure diffusion between fracture and host rock in response to the applied compression.

\section{Characteristics of the fracture normal compliance}

The frequency-dependent fracture normal compliance given by equation 8 has the following low- and high-frequency limits

$$
\lim _{\omega \rightarrow 0} Z_{N}(\omega)=Z_{N}^{\mathrm{nf}}+2 B_{\mathrm{f}} \frac{B_{\mathrm{f}}-B_{\mathrm{hr}}}{\left(N_{\mathrm{f}} / L_{\mathrm{f}}\right)+\left(N_{\mathrm{hr}} / L_{\mathrm{hr}}\right)},
$$

and

$$
\lim _{\omega \rightarrow \infty} Z_{N}(\omega)=Z_{N}^{\mathrm{nf}}
$$

These limits are separated by the so-called characteristic transition frequency $\omega_{c}$ (Brajanovski et al., 2006). For very high frequencies, that is, $\omega \gg \omega_{c}$, the diffusion lengths are very small compared with the thickness of the host rock. Therefore, there is not enough time during an oscillatory half-cycle to equilibrate the induced pressures between the fracture and the host rock. In this unrelaxed case, the fracture normal compliance is real valued and corresponds to that of a thin layer of soft material with WIFF being neglected (equation 10). Conversely, for $\omega \ll \omega_{c}$, the diffusion lengths are much larger than the dimensions of the host rock and, thus, there is enough time for the fluid pressure to equilibrate at a common value. A large amount of fluid flows from the fracture into the host rock during the compression cycle, thus resulting in maximum deformation of the fracture. This, in turn, produces an increase of the solid displacement discontinuity and, consequently, of the fracture compliance. This increase is quantified by the second term of the right side of equation 9. For frequencies close to $\omega_{c}$, the diffusion lengths are comparable with the dimensions of the host rock and, thus, WIFF is significant. The corresponding energy dissipation finds its expression in the solid displacement discontinuity and renders the fracture compliance to a complex quantity. Therefore, expression 8 for the fracture normal compliance encodes wave attenuation and dispersion due to WIFF.

To illustrate the properties of $Z_{N}(\omega)$, we consider a rock model composed of a large number of regularly distributed fractures with a uniform aperture of $0.04 \mathrm{~cm}$. The separation between consecutive fractures is $10 \mathrm{~cm}$. The host rock is a quartz sandstone with a porosity $\phi_{\mathrm{hr}}$ of 0.1 , a solid grain bulk modulus $K_{s}^{\mathrm{hr}}$ of $37 \mathrm{GPa}$, and a grain density $\rho_{s}^{\mathrm{hr}}$ of $2.65 \mathrm{~g} / \mathrm{cm}^{3}$. For the dry frame, we consider a bulk modulus $K_{m}^{\mathrm{hr}}$ of $26 \mathrm{GPa}$, a shear modulus $\mu_{m}^{\mathrm{hr}}$ of $31 \mathrm{GPa}$, and a permeability $\kappa_{\mathrm{hr}}$ of $1 \mathrm{mD}$. We assume that the grain-scale solid material properties of the fracture are identical to those of the host rock. Following Rubino et al. (2014), we use for the dry frame fracture properties $\phi_{\mathrm{f}}=0.9, \quad K_{m}^{\mathrm{f}}=0.024 \mathrm{GPa}, \mu_{m}^{\mathrm{f}}=0.012 \mathrm{GPa}$, and $\kappa_{\mathrm{f}}=$ 100 D. Fractures and host rock are fully saturated with water having a bulk modulus $K_{w}$ of $2.25 \mathrm{GPa}$, a density $\rho_{w}$ of $1.09 \mathrm{~g} / \mathrm{cm}^{3}$, and a shear viscosity $\eta_{w}$ of $0.01 \mathrm{P}$.

Figure 2 shows the fracture normal compliance $Z_{N}(\omega)$ according to equation 8 . We observe that its real part decreases significantly with frequency. Indeed, for the particular physical and geometric characteristics of the materials considered in this study, the normal compliance corresponding to the low-frequency limit is $\sim 16$ times that corresponding to the high-frequency limit, where fluid flow is negligible. In this specific example most of the transition between these two end-member scenarios takes place for frequencies between 1 and $100 \mathrm{~Hz}$, where the magnitude of the imaginary part of $Z_{N}(\omega)$ assumes significant values.

\section{NUMERICAL RECIPE}

\section{Space-frequency domain formulation}

Numerical simulations of seismic wave propagation based on the LST can be implemented in the space-frequency domain (Castromán and Zyserman, 2013), which allows us to readily include complex-valued frequency-dependent fracture normal compliances. To this end, we solve the elastodynamic equation of motion

$$
-\omega^{2} \rho_{b}^{\mathrm{hr}} u_{z}^{\mathrm{hr}}-\frac{\partial \tau_{z z}^{\mathrm{hr}}}{\partial z}=f(z, \omega),
$$

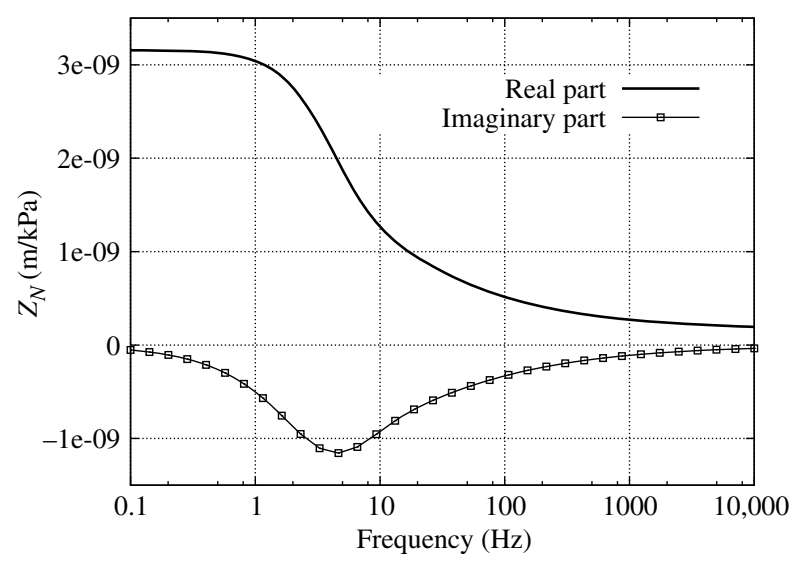

Figure 2. Fracture normal compliance $Z_{N}(\omega)$ for a regular distribution of parallel fractures characterized by an aperture of $0.04 \mathrm{~cm}$ and a separation of $10 \mathrm{~cm}$. 
with $f(z, \omega)$ denoting an external source term and $\rho_{b}^{\mathrm{hr}}, u_{z}^{\mathrm{hr}}$, and $\tau_{z z}^{\mathrm{hr}}$ being the density, vertical displacement, and stress component of the host rock, respectively. We note that by using equation 11 the porous host rock is represented by an equivalent elastic solid. Thus, the bulk density of this elastic medium is given by $\rho_{b}^{\mathrm{hr}}=$ $\phi_{\mathrm{hr}} \rho_{w}+\left(1-\phi_{\mathrm{hr}}\right) \rho_{s}^{\mathrm{hr}}$. Moreover, the stress is related to the displacement by Hooke's law

$$
\tau_{z z}^{\mathrm{hr}}=H_{\mathrm{hr}} \frac{\partial u_{z}^{\mathrm{hr}}}{\partial z},
$$

where $H_{\mathrm{hr}}$ is the plane-wave modulus. Given that we seek a simple way to include WIFF within the LST, we assume that the host rock behaves in an undrained manner independent of the fluid pressure exchange between fracture and host rock. This means that we use Gassmann's (1951) equation to compute $H_{\mathrm{hr}}$. Our modeling recipe is then as follows: The host medium is modeled using equations 11 and 12 . The fractures are replaced by interfaces characterized by

$$
\left[u_{z}^{\mathrm{hr}}\left(z_{j}, \omega\right)\right]=Z_{N}(\omega) \tau_{z z}^{\mathrm{hr}}\left(z_{j}, \omega\right)
$$

with $z_{j}$ denoting the position of the $j$ th fracture and $Z_{N}(\omega)$ being its normal compliance (equation 8). This way, we account for seismic attenuation and dispersion due to WIFF between fractures and the host rock, even though the latter is modeled as an equivalent elastic solid. That is, the use of equation 13 results in the overall model behaving like an effective viscoelastic medium.

\section{Validation for a 1D model of a fractured zone}

To illustrate and validate the proposed extension of the LST, we consider the model shown in Figure 3 consisting of a 700-m-thick sandstone formation with properties similar to those specified in the previous section. At its center, it contains a fractured zone of thickness $E$ having regularly distributed fractures with properties similar to those given above. The separation between consecutive fractures

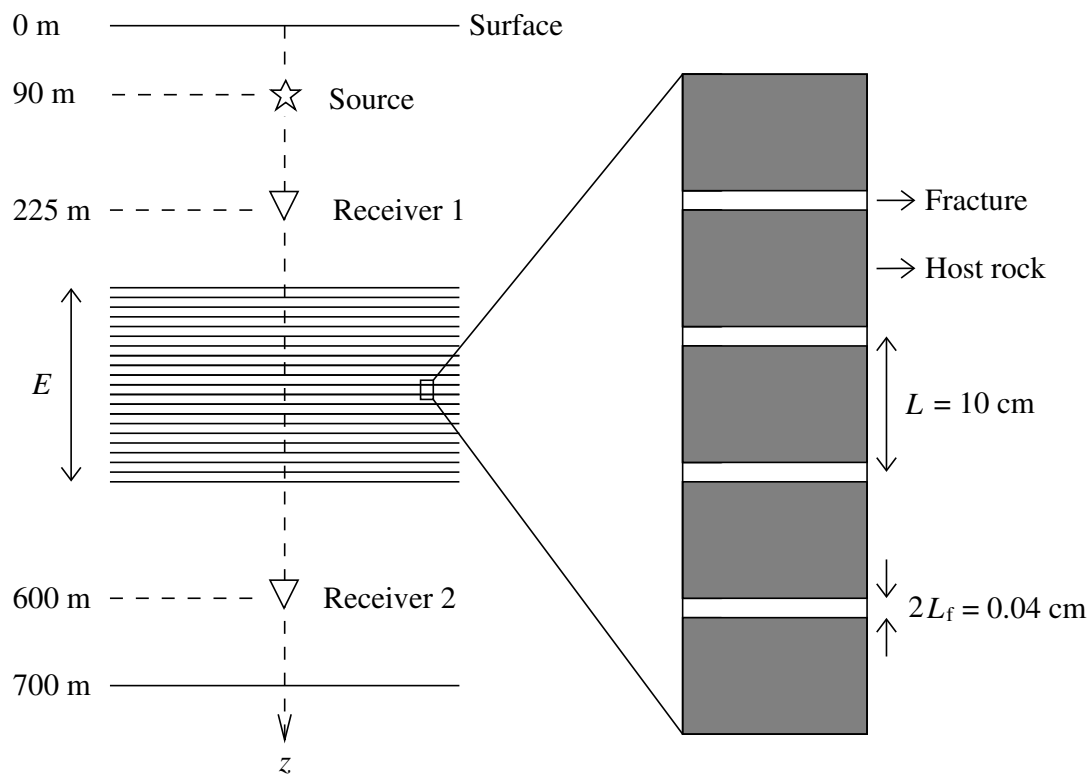

Figure 3. Model setup considered to validate the inclusion of WIFF effects in the LST. The star denotes the depth level of the considered plane-wave source. is $10 \mathrm{~cm}$, and their aperture is of $0.04 \mathrm{~cm}$. The material between the individual fractures is identical to that of the host rock above and below the fractured region and, hence, it is characterized by an undrained P-wave modulus $H_{\mathrm{hr}}=69 \mathrm{GPa}$. Thus, the fracture normal weakness $\Delta_{N}=Z_{N} H_{\mathrm{hr}} /\left(1+Z_{N} H_{\mathrm{hr}}\right)$ ranges from 0.013 at high frequencies to 0.18 at low frequencies. This change of fracture weakness is associated with maximum values of $1 / Q$ of approximately 0.3 . These arguably very high maximum attenuation levels are largely due to the idealized characteristics of the considered fracture set, which consists of a regular distribution of planar fractures of infinite horizontal extension composed of very soft porous material. In the given context, it is, however, important to note that such high attenuation levels serve as an extreme scenario for exploring the overall validity and robustness of the proposed methodology.

To generate synthetic seismograms, we solve equations 11-13 using the discontinuous Galerkin finite-element method (Castromán and Zyserman, 2013). We consider a plane-wave source with a Ricker-type amplitude spectrum centered at $50 \mathrm{~Hz}$, and two receivers recording seismic waves reflected from and transmitted through the fractured zone (Figure 3 ).

To validate our modeling approach, we solve Biot's (1962) propagation equations for the same model setup. That is, we solve

$$
-\omega^{2} \rho_{b} u_{z}-\omega^{2} \rho_{w} w_{z}-\frac{\partial \tau_{z z}}{\partial z}=f_{s}(z, \omega),
$$

$$
-\omega^{2} \rho_{w} u_{z}-\omega^{2} g w_{z}+i \omega \frac{\eta}{\kappa} w_{z}+\frac{\partial p_{f}}{\partial z}=f_{f}(z, \omega)
$$

where $\rho_{b}$ is the bulk density, $g$ is the mass coupling coefficient, and the terms $f_{s}$ and $f_{f}$ compose the external source. The host rock and the fractures are represented as fluid-saturated poroelastic solids. Therefore, WIFF effects are fully accounted for. The governing equations 14 and 15 , coupled through the constitutive relations (equations 5 and 6), are solved by means of a finite-element method, employing $\sim 160$ times the number of degrees of freedom used by the corresponding LST. Four numerical cells are used to represent each fracture to adequately capture WIFF effects. We use absorbing boundary conditions at the edges of the numerical domain for the simulations based on the extension of the LST as well as for those based on Biot's theory.

The black lines in Figure 4 denote the seismic traces recorded at receiver 2 using Biot's propagation equations for different thicknesses of the fractured zone. We observe that transmitted signals experience a strong amplitude decay and pulse broadening as the thickness of the fractured zone increases. This is due to attenuation and velocity dispersion in response to WIFF between the fractures and the host rock. The dots in Figure 4 show the responses obtained with our extension of the LST using the fracture normal compliance $Z_{N}(\omega)$ depicted in Figure 2. Overall, there is very good agreement. This suggests that $Z_{N}(\omega)$ given by equation 8 captures almost all 
attenuation and velocity dispersion experienced by the seismic wave. We do, however, observe that the discrepancies between the results increase with increasing fractured zone thickness, as quantified by the red lines. In particular, we observe that for $E=150 \mathrm{~m}$, the amplitude decay is slightly overestimated and the velocity is underestimated by the LST. These discrepancies are expected because the plane-wave modulus for the host rock is constructed from the undrained bulk modulus given by Gassmann's equation. This means that the host rock plane-wave modulus is constant, whereas WIFF implies a change of the host rock properties.

Figure 5 shows the seismic traces recorded at receiver 1 . The solutions based on Biot's propagation equations indicate that the reflected signal strongly depends on the thickness $E$ of the fractured zone. Indeed, when $E$ is relatively small compared with the seismic wavelength, a single pulse is observed. Conversely, when $E$ is comparable or larger than the wavelength, two pulses are observed which correspond to reflections from the top and bottom boundaries of the fractured zone. The seismogram for $E=150 \mathrm{~m}$ shows strong amplitude decay and pulse broadening for the reflection from the bottom of the fractured zone. We see that for the reflected signals, attenuation and velocity dispersion effects can be successfully included in the LST by using the fracture normal compliance given by equation 8. Again, there are slight discrepancies between the two solutions, as indicated by the red lines. In particular, the amplitudes of the reflections from the top of the fractured zone are overestimated by the LST. This is expected because the phase velocity of the fractured zone is underestimated when using the LST, which in turn produces an overestimation of the acoustic impedance contrast between the fractured zone and the medium above it.

\section{DISCUSSION}

We propose a model for the fracture normal compliance that allows to account for WIFF effects related to the strong compressibility contrasts typically observed between fractures and host rock. In this context, it is important to note that our approach is conceptually different from the work of Nakagawa and Schoenberg (2007), who propose a generalization of the LST to poroelasticity through the derivation of suitable boundary conditions for a single fracture. In contrast, our approach is based on a frequency-dependent complex-valued fracture compliance obtained by solving, either analytically or numerically, a poroelastic boundary value problem for a set of parallel fractures. Thus, the inferred fracture compliance can be included into an LST-based wave propagation algorithm formulated in the space-frequency domain. As such, the proposed methodology can be regarded as a recipe to include poroelastic effects in the LST without the requirement of a poroelastic generalization thereof.

A simplification used in this work is that the 1D fracture system consists of several very thin, highly compliant porous layers oriented perpendicularly to the direction of $\mathrm{P}$-wave propagation. Moreover, the fractures are regularly distributed, and the separation between consecutive fractures is small compared with the prevailing seismic wavelengths which, in turn, implies that elastic scattering effects are not considered in our model. Despite these simplifications, the proposed method is valuable because it is a first step toward the inclusion of WIFF, arguably the predominant seismic energy dissipation mechanism in fractured media, into the LST. This, together with the fact that the LST is the most common theo- retical framework to model the seismic response of fractured media point to the importance of the results shown in this work.

The methodology presented in this study can possibly be generalized to fractures of finite length through the use of 2D numerical oscillatory compressibility tests. These tests would also allow us to explore the effects of different characteristics of the fracture contact areas on the normal compliance (Rubino et al., 2014). Additionally tangential compliances could be obtained by applying oscillatory shear tests to $2 \mathrm{D}$ samples containing horizontal fractures. The resulting parameters would then permit us to account for the effects of contact areas and WIFF associated with both $\mathrm{P}$ - and S-waves impinging

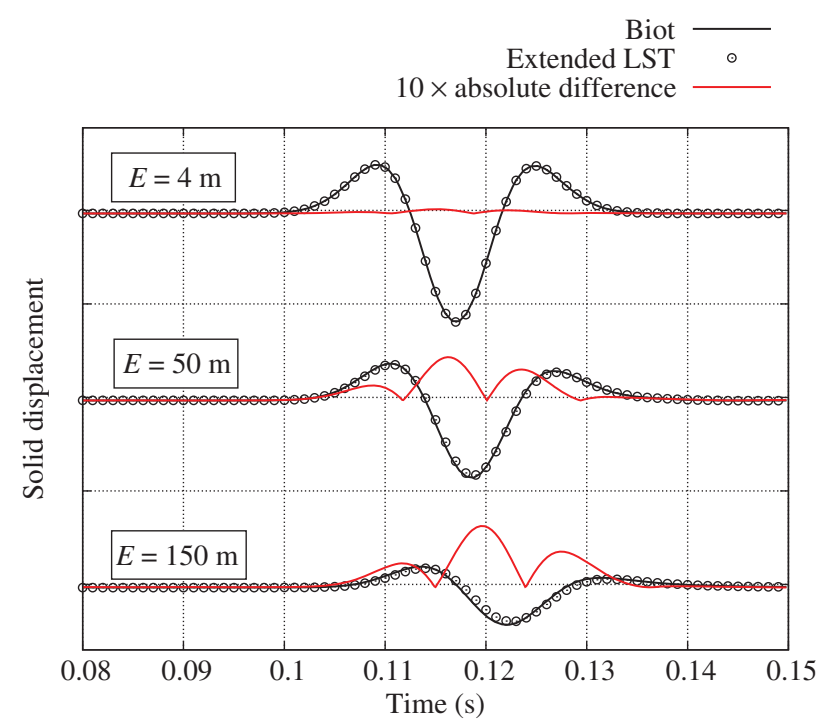

Figure 4. Transmitted signals recorded at receiver 2 obtained using Biot's propagation equations and the extended version of the LST. Red lines indicate 10 times the absolute value of the difference between the two solutions. The model setup is illustrated in Figure 3.

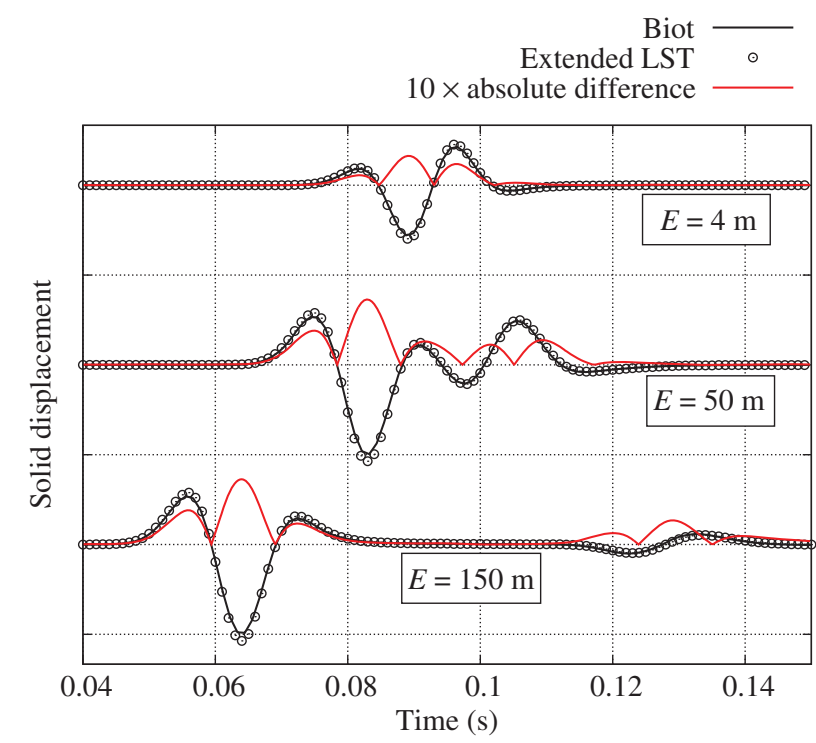

Figure 5. Same as Figure 4, but for seismograms recorded at receiver 1 (reflected signals). 
at different angles on fractured zones. Such 2D oscillatory tests may also permit to compute average complex-valued, frequency-dependent compliances for fractures composing an REV of a reservoir containing irregularly distributed fractures. A potential complication arising in the context of such generalizations is, however, that in these cases, the normal and tangential compliances do not fully determine the properties of fractures embedded in a saturated porous host rock (Gurevich, 2003).

\section{CONCLUSIONS}

We have obtained an analytical expression for a complex-valued and frequency-dependent fracture compliance that allows to include WIFF effects into the LST for a 1D system of regularly distributed fractures. By solving the elastodynamic wave equation in the spacefrequency domain, the thus derived fracture compliance was employed to generate synthetic seismograms for a $1 \mathrm{D}$ model containing a fractured zone embedded in an otherwise homogeneous rock. Comparisons with corresponding simulations based on a numerical solution of the poroelastic equations of motion demonstrate that most of the fairly pronounced WIFF-related seismic attenuation and velocity dispersion characteristics are successfully captured by this extension of the classical LST. Therefore, we conclude that the proposed methodology can be applied to accurately and efficiently generate synthetic seismograms of 1D fractured media, notably in the presence of significant WIFF between the fractures and the host rock.

\section{ACKNOWLEDGMENTS}

This work was supported in part by grants from the Swiss National Science Foundation and the Herbette Foundation of the University of Lausanne.

\section{REFERENCES}

Bakulin, A., V. Grechka, and I. Tsvankin, 2000, Estimation of fracture parameters from reflection seismic data. Part I: HTI model due to a single fracture set: Geophysics, 65, 1788-1802, doi: 10.1190/1.1444863.

Biot, M. A., 1962, Mechanics of deformation and acoustic propagation in porous media: Journal of Applied Physics, 33, 1482-1498, doi: 10.1063/1 .1728759 .

Brajanovski, M., T. M. Müller, and B. Gurevich, 2006, Characteristic frequencies of seismic attenuation due to wave-induced fluid flow in fractured porous media: Geophysical Journal International, 166, 574-578, doi: 10.1111/j.1365-246X.2006.03068.x.
Castromán, G. A., and F. I. Zyserman, 2013, Discontinuous Galerkin methods for solving the acoustic wave equation: Mecánica Computacional, 32, 3905-3918.

Chapman, M., 2003, Frequency-dependent anisotropy due to meso-scale fractures in the presence of equant porosity: Geophysical Prospecting, 51, 369-379, doi: 10.1046/j.1365-2478.2003.00384.x.

Chichinina, T. I., I. R. Obolentseva, and G. Ronquillo-Jarillo, 2009 , Anisotropy of seismic attenuation in fractured media: Theory and ultrasonic experiment: Transport in Porous Media, 79, 1-14, doi: $10.1007 /$ s11242-008-9233-9.

Clark, R. A., P. M. Benson, A. J. Carter, and C. A. Guerrero Moreno, 2009 , Anisotropic P-wave attenuation measured from a multi-azimuth surface seismic reflection survey: Geophysical Prospecting, 57, 835-845, doi 10.1111/j.1365-2478.2008.00772.x.

Coates, R. T., and M. Schoenberg, 1995, Finite-difference modeling of faults and fractures: Geophysics, 60, 1514-1526, doi: 10.1190/1 1443884 .

Gassmann, F., 1951, Über die Elastizität poröser Medien: Vierteljahresschrift der Naturforschenden Gesellschaft in Zürich, 96, 1-23.

Gurevich, B., 2003, Elastic properties of saturated porous rocks with aligned fractures: Journal of Applied Geophysics, 54, 203-218, doi: 10.1016/j .jappgeo.2002.11.002.

Hall, F., and Y. Wang, 2012, Seismic response of fractures by numerica simulation: Geophysical Journal International, 189, 591-601, doi: 10 $.1111 / j .1365-246 X .2012 .05360 . x$.

Liu, E., J. A. Hudson, and T. Pointer, 2000, Equivalent medium representation of fractured rock: Journal of Geophysical Research, 105, 29813000, doi: 10.1029/1999JB900306.

Maultzsch, S., M. Chapman, E. Liu, and X. Y. Li, 2003, Modelling frequency-dependent seismic anisotropy in fluid-saturated rock with aligned fractures: Implication of fracture size estimation from anisotropic measurements: Geophysical Prospecting, 51, 381-392, doi: 10.1046/j $.1365-2478.2003 .00386 . x$.

Nakagawa, S., and M. A. Schoenberg, 2007, Poroelastic modeling of seismic boundary conditions across a fracture: Journal of the Acoustical Society of America, 122, 831-847, doi: 10.1121/1.2747206.

Peacock, S., C. McCann, J. Sothcott, and T. R. Astin, 1994, Experimental measurements of seismic attenuation in microfractured sedimentary rock: Geophysics, 59, 1342-1351, doi: 10.1190/1.1443693.

Pyrak-Nolte, L. J., L. R. Myer, and N. G. W. Cook, 1990, Transmission of seismic waves across single natural fractures: Journal of Geophysical Research, 95, 8617-8638, doi: 10.1029/JB095iB06p08617.

Rubino, J. G., T. M. Müller, M. Milani, and K. Holliger, 2014, Seismic attenuation and velocity dispersion in fractured rocks: The role played by fracture contact areas: Geophysical Prospecting, 62, 1278-1296, doi: 10 $.1111 / 1365-2478.12170$

Schoenberg, M., 1980, Elastic wave behavior across linear slip interfaces: Journal of the Acoustical Society of America, 68, 1516-1521, doi: 10 $.1121 / 1.385077$.

Vlastos, S., E. Liu, I. G. Main, and X. Y. Li, 2003, Numerical simulation of wave propagation in media with discrete distributions of fractures: Effects of fracture sizes and spatial distributions: Geophysical Journal International, 152, 649-668, doi: 10.1046/j.1365-246X.2003.01876.x.

White, J. E., N. G. Mikhaylova, and F. M. Lyakhovitskiy, 1975, Lowfrequency seismic waves in fluid-saturated layered rocks: Physics of the Solid Earth, 11, 654-659.

Worthington, M. H., 2008, Interpreting seismic anisotropy in fractured reservoirs: First Break, 26, 57-63, doi: 10.3997/1365-2397.2008008. 